\title{
Esquemas desadaptativos tempranos y síntomas de depresión en personas atendidas en un hospital especializado en salud mental
}

\author{
Early maladaptive schemas and symptoms of depression in people cared \\ for in a hospital specialized in mental health
}

\author{
Dennys Vanessa Barreda Sánchez-Pachas ${ }^{1}$ \\ Universidad Científica del Sur
}

Recibido: $25-03-21$

Aceptado: $17-05-21$

Publicado: $18-06-21$

\begin{abstract}
Resumen
El objetivo de esta investigación fue establecer la diferencia en la magnitud de los Esquemas Desadaptativos Tempranos (EDT) en muestras clínicas, 136 participantes con síntomas y 135 sin síntomas de depresión, así como evaluar la asociación entre los síntomas de depresión y los EDT. El muestreo fue no probabilístico intencional, contando con 125 hombres y 146 mujeres, atendidas en un hospital especializado en salud mental, previo al diagnóstico. Los instrumentos utilizados fueron: Cuestionario de Esquemas de Young-Versión Breve (YSQS3) y el Inventario de Depresión de Beck II (IDB-II). Los resultados evidenciaron diferencias estadísticamente significativas entre ambos grupos $(p<.05)$ en las cinco dimensiones y sus respectivos esquemas (18), el tamaño de estas fue de pequeñas a grandes, con valores $r_{b i s}$ entre .23 a .64; evidenciando que los esquemas se presentan en mayor magnitud en la muestra con síntomas de depresión. Se confirmó la asociación positiva estadísticamente significativa entre las cinco dimensiones, 17 EDT y los síntomas de depresión, la fuerza de la asociación fue moderada en dos dimensiones y dos EDT y la mínima necesaria para presentar interdependencia entre las variables en tres dimensiones y 15 EDT con coeficientes de determinación que iban de .044 a .383; observándose que los esquemas se presentan más graves en cuanto se presenten síntomas de depresión más intensos; sin embargo esta asociación no se cumplió en el esquema Normas inalcanzables, ya que el plantearse límites inaccesibles, requiere de una alta concentración y exigencia continua lo que no se condice con los síntomas de depresión.
\end{abstract}

Palabras clave: Esquemas desadaptativos tempranos; dimensiones de esquemas; depresión.

\begin{abstract}
The objective of this research was to establish the difference in the magnitude of Early Maladaptive Schemas (EMT) in clinical samples, 136 participants with and 135 without symptoms of depression, as well as to evaluate the association between symptoms of depression
\end{abstract}

1 Docente en la Universidad Científica del Sur. Lima, Perú.

E-mail: paxbonumd@yahoo.es ORCID: https://orcid.org/0000-0002-1927-9190

(C) Los autores. Este artículo es publicado por la Revista de Investigación en Psicología de la Facultad de Psicología, Universidad Nacional Mayor de San Marcos. Este es un artículo de acceso abierto, distribuido bajo los términos de la licencia Creative Commons Atribución 4.0 Internacional (CC BY 4.0) [https://creativecommons.org/licenses/by/4.0/deed.es] que permite el uso, distribución y reproducción en cualquier medio, siempre que la obra original sea debidamente citada de su fuente original. 
and EMT. The sampling was intentional non-probabilistic, with 125 men and 146 women, treated in a hospital specialized in mental health, prior to diagnosis. The instruments used were: Young's Schema Questionnaire-Brief Version (YSQ-S3) and the Beck Depression Inventory II (BDI-II). The results showed statistically significant differences between both groups $(p<.05)$ in the five dimensions and their respective schemas $(18)$, the size of these differences ranged from small to large, with $r_{b i s}$ values between .23 to .64; evidencing that the schemas are presented in greater magnitude in the sample with symptoms of depression. The statistically significant positive association between the five dimensions, 17 EMT and the symptoms of depression was confirmed, the strength of the association was moderate in two dimensions and two EMT and the minimum necessary to present interdependence between the variables in three dimensions and 15 EMT with coefficients of determination ranging from .044 to .383 ; observing that the schemas are more severe as soon as more intense symptoms of depression appear; However, this association is not fulfilled in the Unrelenting standards schema, since considering inaccessible limits requires a high concentration and continuous demand, which is not consistent with the symptoms of depression.

Keywords: Early Maladaptive Schemas; schema domains; depression. 
La calidad de vida de la persona se evidencia en cada una de las áreas en las que ésta se desenvuelve, al alterarse la funcionalidad en estas áreas, dependiendo de la magnitud y la cantidad de áreas afectadas, se altera también la adaptación de las personas. En la actualidad un problema crítico que afecta la calidad de vida a nivel mundial es la depresión (Catalán, 2006; Morales, 2017), según especialistas de la Organización Mundial de la Salud (OMS, 2017, 2020), son 300 millones de personas las afectadas con casos en ascenso cada año y es la primera en ocasionar problemas de salud y discapacidad; asimismo es el principal factor que eleva las estadísticas de los casos de suicidio (Organización Panamericana de la Salud [OPS], 2017). En el Perú, de acuerdo con el equipo de investigadores del Ministerio de Salud (MINSA, 2014, 2020), por 1000 habitantes, se pierden 11.4 años de vida saludables (AVISA), durante el año 2019 en los centros de salud mental comunitarios se atendieron a 250 mil casos de depresión. Al respecto, uno de los enfoques que mayor éxito ha tenido en el tratamiento de la depresión ha sido el cognitivo conductual (Hollon, \& Ponniah, 2010, citados en Carrobles, 2011). A pesar de ello, dentro de la tasa de éxito hay un 30\% de recaídas después de un año de tratamiento (Young et al., 2001, como se citó en Young et al., 2013). Beck (1983) ha trabajado durante años el tema de la depresión, postula que existen esquemas depresivos aprendidos a partir de eventos traumáticos acontecidos durante la infancia y adolescencia, los esquemas para este autor son cogniciones estables sobre las que se generan las percepciones de sí mismo, el mundo y el futuro. Young, discípulo suyo, mejora y amplía los postulados de Beck a partir de los casos clínicos renuentes al cambio (Young et al., 2013). Bajo esta teoría, se conciben los Esquemas desadaptativos tempranos (EDT) como temas generalizados de experiencias dañinas de la infancia que al interrelacionarse con el temperamento del niño se aprenden debido a que, a temprana edad el infante necesita adaptarse a las figuras de apego originarias; sin embargo, estos esquemas en la adultez no permiten que la persona se adapte al contexto generando patrones sobre los que se experimenta cada vivencia, impidiendo así el normal funcionamiento de la persona en la vida adulta (Young et al., 2013).

Los EDT se han generado -como se ha mencionado- a partir del temperamento del niño y de experiencias dañinas, estas podrían deberse a una frustración de las necesidades psicológicas básicas, traumatización o victimización, exceso y distorsión en la satisfacción de las necesidades psicológica básicas y la interiorización selectiva de las figuras parentales como modelos. Las necesidades psicológicas básicas y fundamentales son cinco: Afectos seguros y estables con otras personas, Autonomía, competencia y sentido de identidad, Libertad para expresar necesidades, pensamientos y emociones válidas, Espontaneidad y juego, y finalmente Límites realistas y autocontrol (Young et al., 2013). Al no cubrirse adecuadamente estas necesidades en la infancia generan cinco dimensiones o dominios, cada una de ellos contiene cierto grupo de esquemas que se indican a continuación (véase tabla 1). 
Tabla 1

Dimensiones y Esquemas Desadaptativos Tempranos que las componen

\begin{tabular}{|c|c|c|}
\hline Dimensiones & Esquemas & Creencias Características \\
\hline \multirow[t]{5}{*}{$\begin{array}{l}\text { Desconexión y } \\
\text { rechazo }\end{array}$} & Abandono & $\begin{array}{l}\text { Las figuras significativas no podrán brindar protección y } \\
\text { conexión fiable y estable. }\end{array}$ \\
\hline & Desconfianza y abuso & Los demás dañarán, engañarán o se aprovecharán. \\
\hline & Privación emocional & $\begin{array}{l}\text { Los demás no satisfarán el apoyo emocional, la } \\
\text { protección y la empatía. }\end{array}$ \\
\hline & Imperfección & Sí mismo como defectuoso, sin valía e imperfecto. \\
\hline & $\begin{array}{l}\text { Aislamiento o exclusión } \\
\text { social }\end{array}$ & $\begin{array}{l}\text { Ser notablemente diferente a los demás, dificultad } \\
\text { integrarse al grupo o comunidad. }\end{array}$ \\
\hline \multirow{4}{*}{$\begin{array}{l}\text { Perjuicio en } \\
\text { autonomía y } \\
\text { desempeño }\end{array}$} & Dependencia/ incompetencia & $\begin{array}{l}\text { Ser incapaz de afrontar de manera competente actividades y } \\
\text { compromisos diarios sin el apoyo notable de los otros. }\end{array}$ \\
\hline & Vulnerabilidad & $\begin{array}{l}\text { Una catástrofe externa, enfermedad física o problema } \\
\text { psíquico serán inminentes y no se podrán controlar. }\end{array}$ \\
\hline & Enmarañamiento & $\begin{array}{l}\text { Identidad propia fusionada con otro significativo por apego } \\
\text { excesivo. }\end{array}$ \\
\hline & Fracaso & $\begin{array}{l}\text { Fracaso e ineptitud, en áreas de logro como deportes, lo } \\
\text { académico y/o profesional. }\end{array}$ \\
\hline \multirow{3}{*}{$\begin{array}{l}\text { Dirigido por las } \\
\text { necesidades de los } \\
\text { demás }\end{array}$} & Subyugación & $\begin{array}{l}\text { Los demás se enojarán, lo abandonarán o vengarán si no } \\
\text { cede a sus demandas. }\end{array}$ \\
\hline & Autosacrificio & $\begin{array}{l}\text { Satisfacer las necesidades ajenas en lugar de las propias } \\
\text { para evitar la culpa por el sufrimiento del otro. }\end{array}$ \\
\hline & $\begin{array}{l}\text { Búsqueda de aprobación/ } \\
\text { reconocimiento }\end{array}$ & La estima propia depende de la reacción de los otros. \\
\hline \multirow[t]{4}{*}{$\begin{array}{l}\text { Sobrevigilancia e } \\
\text { inhibición }\end{array}$} & Pesimismo/ Negatividad & $\begin{array}{l}\text { Minimiza los aspectos positivos de la vida, centrando la } \\
\text { atención exageradamente en expectativas negativas. }\end{array}$ \\
\hline & Inhibición emocional & $\begin{array}{l}\text { Control excesivo, de emociones, impulsos y } \\
\text { comportamientos espontáneos. }\end{array}$ \\
\hline & Normas inalcanzables & Perfeccionismo, metas rígidas e irrealmente altas. \\
\hline & Condena o castigo & $\begin{array}{l}\text { Se debe ser sancionado por cometer errores, intolerancia } \\
\text { hacia la falibilidad humana. }\end{array}$ \\
\hline \multirow[t]{2}{*}{ Límites inadecuados } & Grandiosidad & $\begin{array}{l}\text { Superioridad ante los demás, con derechos y facultades } \\
\text { especiales. }\end{array}$ \\
\hline & $\begin{array}{l}\text { Insuficiente autocontrol / } \\
\text { autodisciplina }\end{array}$ & $\begin{array}{l}\text { Busca la gratificación inmediata sin mayor esfuerzo, } \\
\text { impulsivo, intolerante a la frustración. }\end{array}$ \\
\hline
\end{tabular}

Los EDT, están compuestos de cogniciones, memorias, una fuerte carga emocional acompañado de las respectivas sensaciones corporales que se activan cuando la persona percibe un evento como similar a la experiencia dañina vivida en la infancia, a estos eventos que activan los esquemas Young et al. (2013), los ha denominado botones emocionales, por tanto, los EDT no están siempre activos sin embargo, es debido a la llamada química del esquema que la persona se siente atraída de manera inconsciente a circunstancias que tienen en común el tema de la vivencia de origen ya que es así como aprendió desde muy temprana edad a concebirse a sí mismo, sus relaciones con los demás, al mundo y el futuro; al haber automatizado este aprendizaje es que la persona requiere mantener su existencia acorde a como las experimentó en la infancia, esto es la coherencia del esquema, 
es así que aunque en la actualidad sean desadaptativos, los EDT se autoperpetúan según la gravedad de afectación en la calidad de vida.

En las personas con depresión se presentan esquemas (Beck, 1983) que se expresarán de manera particular a través de síntomas tales como la incapacidad de disfrutar actividades que anteriormente sí provocaban placer, apatía, astenia o disminución de la vitalidad y del nivel de actividad con excesivo cansancio o por otro lado agitación psicomotriz difícil de controlar; humor depresivo caracterizado por tristeza, sensación de vacío y desesperanza; pensamientos de culpa, pérdida de la confianza en sí mismo, percepción de inferioridad e inutilidad, dificultad para atender, concentrarse y tomar decisiones, insomnio o hipersomnia, aumento o disminución del apetito, visión negativa del futuro, ideación suicida y disminución acentuada de la libido. (OMS, 1992; Asociación Americana de Psiquiatría [AAP], 2014).

Siguiendo la línea de los EDT, estos están presentes en la población regular, se presentan en todas las personas, pero la magnitud de estos se evidenciará en el perjuicio de la calidad de vida, pudiendo causar diversos trastornos (Young et al., 2013). Estudios en muestras generales (Ribeiro et al., 2014; Cormier et al., 2011) han encontrado que los EDT son más intensos cuanto mayores son los síntomas de depresión, pero son exiguos los estudios con muestras clínicas. Por tanto, es importante ampliar este campo de conocimiento e indicar si hay diferencias incluso dentro de muestras clínicas, así como describir cuáles son los esquemas más activados y su asociación con síntomas de depresión, resulta necesaria una actualización constante sobre este fenómeno, debido a que, a pesar de los esfuerzos por conocer los diferentes determinantes y las acciones para su abordaje (Dirección de Salud Mental del MINSA, 2008; Vásquez, 2016; Pérez-Padilla, et. al, 2017); como se ha detallado, siguen en aumento los casos a nivel mundial; desde el aspecto psicológico, se requiere profundizar sobre los patrones que guían la vida de las personas, como son los EDT. Para obtener mayores datos empíricos que clarifiquen esta descripción es que se desarrolla este estudio a fin de establecer las diferencias entre dos muestras clínicas, una de personas con síntomas de depresión y otra sin síntomas de depresión pero que presentan algún otro problema de salud mental. Así mismo, se plantea la hipótesis de que los esquemas se relacionan directamente con síntomas de depresión.

\section{MÉTODO}

\section{Participantes}

La muestra estuvo constituida por 271 pacientes atendidos en un hospital especializado en salud mental de Lima, los cuales fueron entrevistados en consulta externa en sus primeras visitas al nosocomio, previo a la conclusión diagnóstica de parte del profesional del hospital. El muestreo fue intencional, seleccionado de 
acuerdo a las características y propósitos de la investigación (Hernández et al., 2014). Los criterios de inclusión para la muestra de estudio fueron: ser atendido en consulta externa, disposición para colaborar en la investigación, de ambos sexos y que sus condiciones mentales afectivo-emotivo y cognitivas, así como la orientación en persona, tiempo y espacio, no impidieran comprender y responder los instrumentos.

Los participantes fueron divididos en dos grupos a partir de los resultados obtenidos en el Inventario de Depresión de Beck II (BDI-II), el primer grupo sin síntomas de depresión estuvo constituido por 135 personas que presentaron un nivel ausente o nulo y los participantes que obtuvieron niveles leve, moderado y grave conformaron el grupo con síntomas de depresión, siendo un total de 136 participantes.

Respecto a los datos sociodemográficos, en las personas con síntomas de depresión, se observó que el porcentaje de hombres fue del $44 \%$ mientras que el de mujeres fue de $55.9 \%$, similar distribución a la de la muestra de personas sin síntomas de depresión (Tabla 2), la edad de mayor porcentaje de participantes estuvo comprendida entre los 18 y 29 años en ambos grupos con $52.9 \%$ y $40 \%$ respectivamente; de igual manera en ambos grupos el 55\% era soltero; el $44.9 \%$ y $45.9 \%$ concluyeron sus estudios escolares y el $50 \%$ de personas con síntomas de depresión así como el 59.2\% de personas sin síntomas de depresión se encontraban en actividad laboral.

\section{Instrumentos}

Inventario de Depresión de Beck II (IDB-II), de Beck et al. (2006) en su segunda versión evalúa la intensidad de los síntomas de la depresión durante las dos últimas semanas, cuenta con 21 ítems, cada uno comprende cuatro afirmaciones que van en una escala de 0 (ausente o mínima), hasta 3 (muy intenso o grave). Ha sido adaptado al español por Brenlla y Rodríguez (2006), con un coeficiente alfa de .88; en Perú se confirmaron las propiedades psicométricas del instrumento con niveles elevados de consistencia interna en un alfa de Cronbach de .93 y evidencia de validez basada en el contenido a través del coeficiente V de Aiken, (Penfield y Giacobbi, 2004, como se citó en Merino y Livia, 2009), con valores entre 0.90 a 1.00. Barreda (2019).

Cuestionario de Esquemas de Young-Versión Breve (YSQ-S3), creado por Young (2005), a partir de los ítems con mayor carga representativa de la versión larga. Cada uno de los 90 ítems es evaluado a través de una escala

Likert con seis alternativas, desde 1 (totalmente falso) hasta 6 (me describe perfectamente). El instrumento tiene por objetivo identificar y evaluar la intensidad de los EDT. El YSQ en su versión larga obtuvo un coeficiente alfa de Cronbach con rangos por esquemas entre .83 a .96. La versión breve en el Perú 
para el presente estudio demostró un nivel elevado de consistencia interna de .98 . Asimismo, los valores por esquemas presentaron rangos de fiabilidad entre .72 a 90. La validez de contenido por criterio de jueces alcanzó el 100\% de ítems válidos, con valores V de Aiken entre .66 a .97. (Penfield y Giacobbi, 2004, como se citó en Merino y Livia, 2009).

Tabla 2

Características sociodemográficas

\begin{tabular}{|c|c|c|c|c|}
\hline \multirow{3}{*}{$\begin{array}{c}\text { DATOS } \\
\text { SOCIODEMOGRÁFICOS }\end{array}$} & \multicolumn{4}{|c|}{ MUESTRAS } \\
\hline & \multicolumn{2}{|c|}{ Con Síntomas de Depresión } & \multicolumn{2}{|c|}{ Sin Síntomas de Depresión } \\
\hline & $n=136$ & $\%$ & $n=135$ & $\%$ \\
\hline \multicolumn{5}{|l|}{ Sexo } \\
\hline Hombre & 60 & 44.1 & 65 & 48.1 \\
\hline Mujer & 76 & 55.9 & 70 & 51.9 \\
\hline \multicolumn{5}{|l|}{ Edad } \\
\hline 18 a 29 & 72 & 52.9 & 54 & 40.0 \\
\hline 30 a 39 & 27 & 19.9 & 43 & 31.9 \\
\hline 40 a 49 & 21 & 15.4 & 23 & 17.0 \\
\hline 50 a 59 & 12 & 8.8 & 12 & 8.9 \\
\hline 60 a 71 & 4 & 2.9 & 3 & 2.2 \\
\hline \multicolumn{5}{|l|}{ Estado civil } \\
\hline Casado & 27 & 19.9 & 32 & 23.7 \\
\hline Soltero & 75 & 55.1 & 75 & 55.6 \\
\hline Viudo & 0 & 0 & 2 & 1.5 \\
\hline Divorciado & 8 & 5.9 & 3 & 2.2 \\
\hline Conviviente & 26 & 19.1 & 23 & 17.0 \\
\hline \multicolumn{5}{|l|}{ Grado de instrucción } \\
\hline Escolar incompleta & 18 & 13.2 & 12 & 8.9 \\
\hline Escolar completa & 61 & 44.9 & 62 & 45.9 \\
\hline Superior técnica & 34 & 25.0 & 26 & 19.2 \\
\hline Superior universitaria & 23 & 16.9 & 35 & 26.0 \\
\hline \multicolumn{5}{|l|}{ Ocupación } \\
\hline En casa & 20 & 14.7 & 17 & 12.6 \\
\hline Ama de casa & 15 & 11.0 & 14 & 10.4 \\
\hline Estudiante & 33 & 24.3 & 24 & 17.8 \\
\hline En Actividad laboral & 68 & 50.0 & 80 & 59.2 \\
\hline
\end{tabular}

\section{Procedimiento}

Para la realización de la investigación, previamente se constató el respeto a los principios éticos establecidos a nivel nacional e internacional. Asimismo, se contó con la aprobación del uso de los instrumentos de investigación respetando los derechos de autor. Como paso siguiente se establecieron las coordinaciones 
correspondientes con el jefe del servicio de consulta externa del hospital especializado en salud mental, previa aprobación de la autoridad de la Oficina de Apoyo a la Docencia e Investigación. Luego se estableció el contacto con los participantes, se trabajó con cada uno de ellos de manera individual invitándolos a colaborar de manera voluntaria con la investigación, informándoles acerca del objetivo del estudio y su cualidad anónima y confidencial, firmando un consentimiento escrito. Seguidamente se realizó una entrevista semiestructurada obteniendo información sociodemográfica; se brindaron las instrucciones para responder a los instrumentos lo cual se desarrolló en una sola sesión.

\section{Análisis de datos}

Se procesaron los datos a través el programa SPSS 24.0. Como primer paso se realizaron los análisis psicométricos de los dos instrumentos con su respectiva confiabilidad y validez. En segundo lugar, se realizaron los análisis descriptivos de las variables a través de la bondad de juste a la curva normal, mediante la prueba de Shapiro-Wilk. Finalmente, el análisis inferencial se realizó adoptando el $5 \%$ de nivel de significancia estadística en los estadísticos $U$ de Mann-Whitney y Correlación de Spearman; se trabajó la magnitud del efecto primero para evaluar el tamaño de las diferencias entre los grupos a través del coeficiente de correlación biserial y luego para evaluar la fuerza de asociación entre las variables con el coeficiente de determinación.

\section{RESULTADOS}

Los datos para las variables depresión y esquemas (EDT), se distribuyeron de manera asimétrica, habiéndose evaluado a través del estadístico Shapiro-Wilk y un $p$ valor inferior al nivel de significancia $p<.05$ en la variable depresión y en los 18 esquemas con sus respectivas dimensiones.

A continuación se muestran los valores de las medias en cada grupo, todos los valores promedio en el grupo con síntomas de depresión fueron más altos que el grupo sin depresión, en las personas con síntomas de depresión los cinco esquemas con mayor magnitud fueron Abandono, Búsqueda de aprobación, Insuficiente autocontrol, Normas inalcanzables y Grandiosidad, mientras que en el segundo grupo, los cinco esquemas con mayor valor promedio fueron Normas inalcanzables, Búsqueda de aprobación, Autosacrificio, Abandono y Grandiosidad, compartiendo cuatro esquemas con mayor promedio resaltados en negrita en la Tabla 3.

Para establecer las diferencias entre los grupos se utilizó el estadístico $U$ de Mann-Whitney para muestras independientes, como se observa en la tabla 4, se obtuvieron valores $p$ inferiores al nivel de significancia, $p<.001$, lo que indica que existen diferencias estadísticamente significativas en todas las dimensiones y sus 
respectivos esquemas, entre las muestras de personas con y sin síntomas de depresión atendidas en un centro especializado en salud mental.

Tabla 3

Valores promedio de los esquemas en personas con y sin sintomas de depresión

\begin{tabular}{lcllc}
\hline \multicolumn{1}{c}{ Con Síntomas de Depresión } & \multicolumn{1}{c}{ Sin Síntomas de Depresión } \\
EDT & $\boldsymbol{M}$ & \multicolumn{1}{c}{ EDT } & $\boldsymbol{M}$ \\
\hline Abandono & $\mathbf{1 9 . 2 4}$ & Normas inalcanzables & $\mathbf{1 4 . 4 8}$ \\
Búsqueda de aprobación & $\mathbf{1 8 . 7 6}$ & Búsqueda de aprobación & $\mathbf{1 3 . 9 5}$ \\
Insuficiente autocontrol & 18.35 & Autosacrificio & 12.94 \\
Normas inalcanzables & $\mathbf{1 8 . 2 9}$ & Abandono & $\mathbf{1 2 . 8 1}$ \\
Grandiosidad & $\mathbf{1 8 . 1 0}$ & Grandiosidad & $\mathbf{1 2 . 8 1}$ \\
Desconfianza y abuso & 17.79 & Desconfianza y abuso & 12.48 \\
Negatividad & 17.78 & Negatividad & 12.21 \\
Inhibición emocional & 17.63 & Inhibición emocional & 12.14 \\
Privación emocional & 17.45 & Castigo & 12.05 \\
Vulnerabilidad & 17.03 & Insuficiente autocontrol & 11.87 \\
Dependencia & 16.68 & Vulnerabilidad & 11.44 \\
Exclusión social & 16.42 & Privación emocional & 10.84 \\
Castigo & 16.24 & Enmarañamiento & 10.33 \\
Autosacrificio & 16.04 & Exclusión social & 10.10 \\
Fracaso & 15.85 & Subyugación & 9.61 \\
Subyugación & 15.78 & Dependencia & 9.42 \\
Enmarañamiento & 14.60 & Fracaso & 8.85 \\
Imperfección & 14.38 & Imperfección & 8.02 \\
\hline
\end{tabular}

Nota: Con Síntomas de Depresión $n=136$. Sin Síntomas de Depresión $n=135$. En negrita se muestran los esquemas con medias más altas compartidos en ambos grupos.

Seguidamente se evaluó el tamaño del efecto para evaluar la magnitud de las diferencias entre ambas muestras (Domínguez, 2018). Observándose diferencias de magnitud grande con un coeficiente de correlación biserial entre $r_{b i s}=.64$ y $r_{b i s}=.52$ en la variable esquemas y las dimensiones a excepción de Dirigido por las necesidades de los demás $r_{b i s}=.48$ en la que se obtuvo una magnitud del efecto mediano. Respecto a los esquemas, la magnitud de la diferencia fue grande en seis de ellos: Imperfección, Dependencia, Fracaso, Subyugación, Negatividad e Insuficiente Autocontrol; 11 esquemas presentaron diferencias medianas y finalmente un esquema, Autosacrificio presentó una diferencia de magnitud pequeña, como se muestra en la Tabla 4. 
Tabla 4

Prueba de Muestras Independientes para los EDT y sus Dimensiones

\begin{tabular}{|c|c|c|c|c|c|}
\hline Esquemas y Dominios de Esquemas & $\begin{array}{l}\text { Ude Mann- } \\
\text { Whitney }\end{array}$ & $\begin{array}{c}\text { Con Síntomas de } \\
\text { Depresión Rango } \\
\text { promedio } \\
(n=136)\end{array}$ & $\begin{array}{l}\text { Sin Síntomas de } \\
\text { Depresión } \\
\text { Rango promedio } \\
(n=135)\end{array}$ & $\begin{array}{c}\text { Valor } \\
\text { de } p\end{array}$ & $r_{b i s}$ \\
\hline Esquemas disfuncionales tempranos & 2466.50 & 185.36 & 86.27 & .000 & .63 \\
\hline Dimensión desconexión y rechazo & 2879.00 & 182.33 & 89.33 & .000 & .59 \\
\hline $\begin{array}{l}\text { Dimensión perjuicio en autonomía y } \\
\text { desempeño }\end{array}$ & 2409.00 & 185.79 & 85.84 & .000 & .64 \\
\hline $\begin{array}{l}\text { Dimensión dirigido por las necesidades } \\
\text { de los demás }\end{array}$ & 4103.50 & 173.33 & 98.40 & .000 & .48 \\
\hline Dimensión sobrevigilancia e inhibición & 3656.00 & 176.62 & 95.08 & .000 & .52 \\
\hline Dimensión límites inadecuados & 3095.00 & 180.74 & 90.93 & .000 & .57 \\
\hline Abandono & 4324.00 & 171.71 & 100.03 & .000 & .46 \\
\hline Desconfianza & 5008.00 & 166.68 & 105.10 & .000 & .39 \\
\hline Privación Emocional & 4066.50 & 173.60 & 98.12 & .000 & .48 \\
\hline Imperfección & 3579.50 & 177.18 & 94.51 & .000 & .53 \\
\hline Exclusión Social & 4526.00 & 170.22 & 101.53 & .000 & .44 \\
\hline Dependencia & 3125.50 & 180.52 & 91.15 & .000 & .57 \\
\hline Vulnerabilidad & 4204.00 & 172.59 & 99.14 & .000 & .47 \\
\hline Enmarañamiento & 5228.50 & 165.06 & 106.73 & .000 & .37 \\
\hline Fracaso & 3492.00 & 177.82 & 93.87 & .000 & .54 \\
\hline Subyugación & 3260.50 & 179.53 & 92.15 & .000 & .56 \\
\hline Autosacrificio & 6726.00 & 154.04 & 117.82 & .000 & .23 \\
\hline Búsqueda de Aprobación & 5208.00 & 165.21 & 106.58 & .000 & .37 \\
\hline Negatividad & 3864.50 & 175.08 & 96.63 & .000 & .50 \\
\hline Inhibición Emocional & 4869.50 & 167.69 & 104.07 & .000 & .41 \\
\hline Normas Inalcanzables & 5460.50 & 163.35 & 108.45 & .000 & .35 \\
\hline Castigo & 5593.00 & 162.38 & 109.43 & .000 & .34 \\
\hline Grandiosidad & 4337.50 & 171.61 & 100.13 & .000 & .46 \\
\hline Insuficiente Autocontrol & 3172.00 & 180.18 & 91.50 & .000 & .57 \\
\hline
\end{tabular}

Nota: $p<.05$

Para la contrastación de la hipótesis en la que se sostiene que los esquemas se asocian con síntomas de depresión, se utilizó el estadístico de Correlación de Spearman, y aunque este mismo estadístico es en sí mismo una medida del tamaño del efecto se trabajó con el coeficiente de determinación de acuerdo con Ferguson (2009), para evaluar la fuerza de la asociación. Se observó que existe una correlación estadísticamente significativa y positiva $(p<.05)$ en los esquemas Subyugación $\left(\mathrm{r}_{\mathrm{s}}=.619\right)$ e Insuficiente autocontrol $\left(\mathrm{r}_{\mathrm{s}}=.498\right)$, la magnitud del efecto fue de $\mathrm{r}_{\mathrm{s}}{ }^{2}=.383 \mathrm{y} \mathrm{r}_{\mathrm{s}}{ }^{2=} .248$ respectivamente, indicando una fuerza media; de 
igual modo las dimensiones, Desconexión y rechazo $\left(\mathrm{r}_{\mathrm{s}}=.539, \mathrm{r}_{\mathrm{s}}^{2}=.291\right)$ así como Perjuicio en autonomía y desempeño $\left(\mathrm{r}_{\mathrm{s}}=.610, \mathrm{r}_{\mathrm{s}}^{2}=.372\right)$. Se obtuvo una fuerza mínima de asociación en las correlaciones directas estadísticamente significativas de las dimensiones Dirigido por las necesidades de los demás $\left(\mathrm{r}_{\mathrm{s}}=.470, \mathrm{r}_{\mathrm{s}}^{2}=.221\right)$, Sobrevigilancia e inhibición $\left(\mathrm{r}_{\mathrm{s}}=.350, \mathrm{r}_{\mathrm{s}}^{2}=.123\right)$ y Límites inadecuados $\left(\mathrm{r}_{\mathrm{s}}=.466, \mathrm{r}_{\mathrm{s}}^{2}\right.$ $=.217$ ), así como en 15 esquemas con valores que van desde $\mathrm{r}_{\mathrm{s}}=.210, \mathrm{r}_{\mathrm{s}}{ }^{2}=.044$ hasta $\mathrm{r}_{\mathrm{s}}=.480, \mathrm{r}_{\mathrm{s}}^{2}=.230$, como se muestra en la Tabla 5; finalmente, no se evidenciaron correlaciones significativas ni una fuerza mínima necesaria en el EDT Normas inalcanzables $\left(\mathrm{r}_{\mathrm{s}}=.073, \mathrm{r}_{\mathrm{s}}^{2}=.005\right)$.

Tabla 5

Correlación entre EDT y síntomas de depresión

\begin{tabular}{|c|c|c|c|}
\hline Esquemas y Dominios de Esquemas & $\begin{array}{c}\text { Coeficiente de } \\
\text { Correlación }\end{array}$ & $\begin{array}{l}\text { Coeficiente de } \\
\text { Determinación }\end{array}$ & Valor de $p$ \\
\hline Esquemas disfuncionales tempranos & $.625^{* *}$ & .391 & .000 \\
\hline Dimensión desconexión y rechazo & $.539 * *$ & .291 & .000 \\
\hline Dimensión perjuicio en autonomía y desempeño & $.610^{* *}$ & .372 & .000 \\
\hline Dimensión dirigido por las necesidades de los demás & $.470 * *$ & .221 & .000 \\
\hline Dimensión sobrevigilancia e inhibición & $.350 * *$ & .123 & .000 \\
\hline Dimensión límites inadecuados & $.466^{* *}$ & .217 & .000 \\
\hline Abandono & $.458^{* *}$ & .210 & .000 \\
\hline Desconfianza y Abuso & $.370 * *$ & .137 & .000 \\
\hline Privación Emocional & $.254 * *$ & .065 & .003 \\
\hline Imperfección & $.438 * *$ & .192 & .000 \\
\hline Exclusión Social & $.392 * *$ & .154 & .000 \\
\hline Dependencia & $.458^{* *}$ & .210 & .000 \\
\hline Vulnerabilidad & $.356^{* *}$ & .127 & .000 \\
\hline Enmarañamiento & $.354 * *$ & .125 & .000 \\
\hline Fracaso & $.480 * *$ & .230 & .000 \\
\hline Subyugación & $.619 * *$ & .383 & .000 \\
\hline Autosacrificio & $.271 * *$ & .073 & .001 \\
\hline Búsqueda de Aprobación & $.226^{* *}$ & .051 & .008 \\
\hline Negatividad & $.446^{* *}$ & .199 & .000 \\
\hline Inhibición Emocional & $.272 * *$ & .074 & .001 \\
\hline Normas Inalcanzables & .073 & .005 & .400 \\
\hline Castigo & $.210^{*}$ & .044 & .014 \\
\hline Grandiosidad & $.307^{* *}$ & .094 & .000 \\
\hline Insuficiente Autocontrol & $.498 * *$ & .248 & .000 \\
\hline
\end{tabular}

Nota: $N=136$, Correlación de Spearman, **. La correlación es significativa en el nivel 0,01 (bilateral). *. La correlación es significativa en el nivel 0,05 (bilateral). 


\section{DISCUSIÓN}

En esta investigación se observa que las personas con síntomas de depresión presentan en mayor magnitud todos los 18 esquemas y sus dimensiones, a diferencia de la otra muestra clínica que no presenta síntomas de depresión. Aunque en ambas muestras los EDT se encuentran activados (Young et al., 2013), en las personas con síntomas de depresión estos se muestran aún más rígidos y extremos; la diferencia significativa entre ambas muestras clínicas, evidencia que la intensidad de los EDT en personas con síntomas de depresión, afecta mayor número de vivencias y áreas de desenvolvimiento, estos datos brindan un mayor aporte a investigaciones anteriores que habían observado que problemas de salud mental como la depresión se relacionan con una menor calidad de vida en diferentes grupos etarios (Ornelas Ramírez \& Ruíz Martínez, 2017; Flores et al., 2012), además de las bajas en productividad laboral (OMS, 2019). La diferencia entre las muestras se puede explicar por la comorbilidad que se presenta frecuentemente en enfermedades físicas crónicas (Merino-Martínez et al., 2019; Rivarola et al., 2019), así como con otros problemas psíquicos tales como ansiedad generalizada, trastorno de pánico y trastorno de somatización (Aragonés et al., 2009; OMS, 2004; OPS, 2017). Resulta necesario y urgente el trabajo en programas efectivos de promoción y prevención en salud mental, tomando en cuenta que en el presente estudio el mayor grupo etario se encuentra entre los 18 a 29 años (52.9\%); el 50\% de los participantes se encuentra en actividad laboral y el $24 \%$ es estudiante.

Respecto a la hipótesis que sostiene que existen correlaciones entre los EDT y la depresión, los datos empíricos de esta investigación confirman que existe asociación positiva significativa en todas las dimensiones y esquemas a excepción de Normas inalcanzables, lo que se explicará a continuación.

Se observan diferencias significativas en la primera dimensión Desconexión y rechazo, en esta y en el esquema Imperfección esta diferencia es grande y moderada en los otros cuatro EDT, indicando que si bien las personas sin síntomas de depresión también presentan esquemas activados, en las personas con síntomas de depresión la necesidad de afecto seguro y estable se encuentra menos satisfecha, sin una figura de apego que le brindara atención, comprensión, cuidado, protección y amor incondicional, cruciales en la infancia (García et al., 2016); por lo que en la actualidad presentan mayores dificultades que otros pacientes en generar vínculos estables y que promuevan un bienestar mutuo. Es de crucial importancia trabajar desde los primeros años de vida para satisfacer un apego seguro y estable, de lo contrario se podría ir gestando la depresión, Durbin et al., (2000) mostraron que ambientes adversos en el hogar son los mayores predictores de un trastorno depresivo a largo plazo.

Siguiendo en la dimensión Desconexión y rechazo, hay una asociación positiva, moderada y significativa entre esta dimensión o dominio y los síntomas de 
depresión, mientras que en los EDT que la componen esta asociación es la mínima necesaria; indicando que la satisfacción de un apego seguro y estable con las figuras significativas son vitales durante la infancia y cuya falencia repercute notoriamente en la calidad de los vínculos con los otros significativos, así por ejemplo Santelices et al. (2011) mostraron que el apego inseguro en adultos se asocia con la depresión. Es así que en el esquema de Abandono, los participantes con síntomas de depresión se caracterizan por presentar una fuerte percepción de soledad perpetua; en el EDT Desconfianza y abuso, lamentan que no haya persona en quien confiar pues tarde o temprano los otros los dañarán, Jimeno (2015) mostró que menores que percibieron carencias en el afecto presentan mayores dificultades en adaptación a nivel personal y social, y aquellos que han sufrido maltrato (como en el esquema desconfianza y abuso) son más propensos a desarrollar depresión que aquellos que no han sufrido maltrato; por la Imperfección, presentan la convicción de que su existencia es fallida y si alguien llegara a conocerlos realmente no los aceptarán; por la Exclusión social, suelen aislarse por no sentirse parte de grupo alguno.

En la dimensión Perjuicio en Autonomía y desempeño, así como los esquemas Dependencia y Fracaso la diferencia es grande entre las muestras de estudio y moderada en los otros dos EDT que componen esta dimensión; ello indica que las personas con síntomas de depresión vieron frustrada desde temprana edad su capacidad para desenvolverse por sus propios recursos y ser reforzados en sus propias habilidades en detrimento del desarrollo de su propia identidad.

$\mathrm{Al}$ evaluar la correlación, se observa una asociación moderada entre esta dimensión con los síntomas de depresión, y una correlación mínima en cada esquema, datos que coinciden con los de Ribeiro et al. (2014) en muestras generales y Renner et al. (2011) en muestras clínicas, es así que en el EDT Dependencia presentan mayores dificultades en tomar decisiones por sí mismos, en Vulnerabilidad se perciben frágiles e indefensos con ansiedad constante ante inminentes desgracias, en Enmarañamiento, hay una fusión emocional entre el paciente y una figura significativa; en Fracaso, no creen poder obtener logros en ninguna área de rendimiento.

En relación a la tercera dimensión Dirigido por las necesidades de los demás, se observa una diferencia moderada, esto indica que las personas con síntomas de depresión, presentaron mayores dificultades en el ambiente de su infancia para poder expresar sus emociones, opinar, elegir e incluso discrepar con respeto, que el grupo sin depresión. Por otro lado, la asociación, aunque es mínima, implica que en la actualidad presentan dificultades en mantener vínculos en los que desdeña sus propias necesidades.

Respecto al EDT Subyugación, presenta una diferencia grande respecto a la muestra clínica sin síntomas de depresión, así como una correlación moderada con los síntomas de depresión, debido a que han aprendido desde pequeños a someterse ante figuras que considera de mayor jerarquía o más poderosas con la finalidad de 
evitar el enfado, la crítica negativa o rechazo, hallazgos encontrados también en jóvenes de muestras generales por Florenzano et al., (2009), estas características se asocian fuertemente con los síntomas de depresión tales como la pérdida de confianza en sí mismo y sentimientos de inferioridad. En el esquema Autosacrificio, la diferencia significativa entre las muestras es pequeña y presenta una mínima correlación con los síntomas de depresión, por este EDT se tiende a poner en primer lugar las necesidades de los otros con la finalidad de que estos no sufran, pero guardan rencor por no recibir de los otros algo a cambio de su sacrificio; con el esquema de Búsqueda de aprobación, se observa una diferencia moderada respecto al grupo de pacientes sin síntomas de depresión debido a que las personas con síntomas de depresión presentan en mayor magnitud una autoestima dependiente de las reacciones de los demás, asimismo hay correlación que -aunque de fuerza baja- entre la magnitud de la depresión y buscar ser aceptados, queridos o admirados por los otros.

En la Dimensión sobrevigilancia e inhibición, hay diferencia grande entre las muestras investigadas, ello indica que los entornos tempranos de las personas con síntomas de depresión presentaron mayores falencias en permitir a los niños ser espontáneos, jugar y descubrir sus propios intereses y preferencias. La correlación es la mínima necesaria para establecer la interdependencia con los síntomas de depresión en los EDT de esta dimensión, de modo que en la actualidad hay un deterioro de la capacidad para permitirse un respiro en sus actividades y disfrutar de sus vivencias por la presión constante de sus distorsiones cognitivas. Es así que la asociación significativa con el EDT Negatividad o Pesimismo, síntoma propio de la depresión, hace referencia a la visión sombría de futuro y de los demás; así como en Castigo, hay una percepción rígida de una grave sanción para sí mismo y los demás por los errores cometidos; en Inhibición emocional el sujeto se muestra reprimido, limitado y plano emocionalmente por vergüenza o temor al rechazo por como expresa sus emociones. En relación al esquema Normas Inalcanzables se observa que es el único EDT en el que no se confirman correlaciones significativas ni con la mínima fuerza esperada; en una muestra de población general Caputto, Cordero, Keegan y Arana, (2015) encontraron que este esquema se asocia con el perfeccionismo tanto en sus vertientes adaptativas como desadaptativas. El valor promedio alcanzado en este esquema es alto al estar entre los cinco primeros en la muestra, aunque se encuentra activado no se puede aceptar la asociación con la depresión, ya que es un esquema en el que la persona requiere concentrarse, dirigir su motivación y voluntad a cumplir estándares muy altos y rigurosos, a nivel social, económico, moral o académico; será importante continuar investigando al respecto, en un mundo en donde se considera la alta competitividad y la sobre exigencia como un factor positivo sin observar las consecuencias a mediano o largo plazo en la salud de la persona y el deterioro que puede ocasionar en otras áreas de su vida como en el distrés y la propia depresión al generar posteriormente desesperanza por no alcanzar sus estándares autoimpuestos. 
Finalmente, en la dimensión Límites inadecuados, hay una diferencia grande entre los participantes con y sin síntomas de depresión; específicamente en el esquema de Grandiosidad esta diferencia es moderada mientras que en Insuficiente autocontrol la diferencia es grande; ello hace referencia a que ambos grupos presentaron falencias en aprender límites adecuados a sus acciones y desarrollar autocontrol; pero las personas con síntomas de depresión presentan mayores dificultades en ser recíprocos, respetuosos de las normas y alcanzar objetivos a largo plazo, ya que desean gratificaciones inmediatas. Por otro lado, existe correlación significativa positiva y mínima en este dominio, así como en el esquema Grandiosidad, mientras que en el esquema Insuficiente autocontrol se observa correlación positiva media, indicando que las personas no aprendieron a ser tolerantes a la frustración y reaccionan con prepotencia, pudiendo ser muestra de un síntoma de la irritabilidad de la depresión y una manera de acallar sensación de vacío y desesperanza que provienen de otros esquemas como Abandono. Los esquemas de Insuficiente autocontrol y el de Subyugación fueron los que mayor fuerza de asociación mostraron con los síntomas de depresión, aunque ésta fue moderada, será importante continuar investigando sobre estos, así como las necesidades psicológicas básicas insatisfechas que generan las dimensiones o dominios que contienen a estos EDT, de manera que el abordaje terapéutico y preventivo-promocional pueda ser más efectivo, mejorando la salud mental de la población.

La interinfluencia entre las variables de estudio muestra que la depresión se mantiene debido a los EDT subyacentes; y los esquemas a su vez se autoperpetúan por los síntomas de depresión como pensamientos y otras conductas disfuncionales ante diversas vivencias que no permiten que la persona se adapte al entorno.

Dentro de las limitaciones se encuentran el ser un estudio descriptivo correlacional por lo que no se puede considerar relaciones causales, así mismo al realizarse la evaluación en la fase inicial a consulta externa no se ha podido tener los diagnósticos específicos de la muestra sin síntomas de depresión. Sin embargo, se abren posibilidades para investigar la presencia de esquemas en diferentes diagnósticos, así como evaluar por etapas de tratamiento.

\section{CONCLUSIONES}

En esta investigación se observó que los 18 EDT y sus respectivas dimensiones presentan diferencias significativas en su magnitud en muestras clínicas de personas con síntomas de depresión quienes presentaron mayor intensidad en la activación de los esquemas a diferencia de personas con otros problemas psicológicos.

Se observó también que los síntomas de depresión presentan una relación directa con las cinco dimensiones y 17 esquemas, con fuerza de asociación moderada en los esquemas de Subyugación e Insuficiente autocontrol. Es decir, que los 
pacientes presentan mayor intensidad en los síntomas de depresión en cuanto más activados y críticos se presenten los esquemas y viceversa.

Por otro lado, no se observa una relación significativa ni mínima con el EDT Normas inalcanzables en el que la persona se caracteriza por presentar una exigencia muy alta de lograr la perfección que nunca se alcanza, el mantener el esfuerzo constante demanda de los propios recursos, pero en las personas con síntomas de depresión, la motivación, concentración y voluntad se presentan endebles.

\section{REFERENCIAS}

Aragonés, E., Lluís Piñol, J., \& Labad A. (2009). Comorbilidad de la depresión mayor con otros trastornos mentales comunes en pacientes de atención primaria. Atención Primaria, 4l(10), 545-551. https://doi:10.1016/j.aprim.2008.11.011

Asociación Americana de Psiquiatría (2014). Guía de consulta de los criterios diagnósticos del DSMV. (5 ${ }^{\text {a }}$ ed.).

Barreda, D. (2019). Propiedades psicométricas del Inventario de Depresión de Beck-II (IDB-II) en una muestra clínica. Revista de Investigación en Psicología, 22(1), 39-52. https://doi.org/10.15381/rinvp.v22i1.16580

Beck, A. (1983). Terapia cognitiva de la depresión. Desclee de Brouwer.

Beck, A. T., Steer, R. A., \& Brown, G. K. (2006). BDI-II. Inventario de Depresión de Beck. Paidós

Brenlla, M. E., \& Rodríguez, C. M. (2006). Adaptación argentina del Inventario de Depresión de Beck (BDI-II). En A. T. Beck, R. A. Steer y G. K. Brown (Ed.), BDI-II. Inventario de Depresión de Beck. (pp. 11-38). Paidós.

Caputto, I., Cordero, S., Keegan, E., \& Arana, F. (2015). Perfeccionismo y esquemas desadaptativos tempranos: Un estudio con estudiantes universitarios. Ciencias Psicológicas 9(2), 245-257. http://www.scielo.edu.uy/scielo.php?script=sci_arttext\&pid $=\mathrm{S} 1688-42212015000300003$

Carrobles, J. (2011). Trastornos depresivos. En V. Caballo, I. Caro \& J. Carrobles (Eds.), Manual de psicopatología y trastornos psicológicos (pp. 311-334). Pirámide.

Catalán, R. (2006). Depresión: Factores Psicosociales. En G. L. Oblitas. (Ed.), Psicología de la salud y enfermedades crónicas (pp.227- 237). Psicom Editores.

Cormier, A., Jourda, B., Laros, C., Walburg, V., \& Callahan, S. (2011). L'influence entre les schémas précoces inadaptés et la dépression [La influencia entre los esquemas desadaptativos tempranos y la depresión]. L'encéphale, 37(4), 293-298. https://doi: 10.1016/j.encep.2011.01.001

Dirección de Salud Mental del MINSA. (2008). Guias de práctica clínica en salud mental y psiquiatría. Guía de Práctica Clínica en Depresión. http://bvs.minsa.gob.pe/local/ MINSA/1756.pdf 
Domínguez, S. (2018). Magnitud del efecto: Una guía rápida. Educación Médica, 19(4), 251 - 254. doi: 10.1016/j.edumed.2017.07.002

Durbin, C.E., Klein, D.N., \& Schwartz, J.E. (2000). Predicting the 2 1/2 year outcome of dysthymia disorder: The roles of childhood adversity and family history of psychopathology [Prediciendo el resultado a los dos años y medio del trastorno de distimia: Los roles de la adversidad infantil y los antecedentes familiares de psicopatología]. Consulting and Clinical Psychology, 68(1), 57-63. https://doi: 10.1037//0022-006X.68.1.57

Ferguson, C. J. (2009). An effect size primer: A guide for clinicians and researchers. [Una cartilla del tamaño del efecto: Una guía para médicos e investigadores]. Professional Psychology: Research and Practice, 40(5), 532-538. https://doi.org/10.1037/a0015808

Florenzano, R., Valdés, M., Cáceres, E., Casassus, M., Sandoval, A., Santander, S., \& Calderón, S. (2009). Percepción de la relación parental entre adolescentes mayores y menores de 15 años. Revista Chilena de Pediatría, 80(6), 520-527. http://www.scielo. $\mathrm{cl} / \mathrm{pdf} / \mathrm{rcp} / \mathrm{v} 80 \mathrm{n} 6 / \mathrm{art04} . \mathrm{pdf}$

Flores, M. E., Cervantes, G. A., González, G. J., Vega, M. G., \& Valle, M. A. (2012). Ansiedad y depresión como indicadores de calidad de vida en adultos mayores. Revista de Psicología da IMED, 4(1), 649-661. https://www.researchgate.net/publication/258499943_ANSIEDAD_Y_DEPRESION_COMO_INDICADORES_DE_CALIDAD_DE_VIDA_EN_ADULTOS_MAYORES

García, N., Rodríguez, E., Duarte, L., \& Bermúdez, M. (2016). Las prácticas de crianza y su relación con el vínculo afectivo. Revista iberoamericana de psicología: ciencia y tecnología, 9(2), 113-124. https://dialnet.unirioja.es/servlet/articulo?codigo=6124351

Hernández, R., Fernández, C., \& Baptista, P. (2014). Metodología de la Investigación. McGraw- Hill.

Jimeno, M. (2015). Experiencias traumáticas en la infancia y su influencia sobre el desarrollo afectivo-social y la memoria autobiográfica en adolescentes institucionalizados. [Tesis doctoral Universidad de Castilla-La Mancha]. Repositorio de la Universidad de Castilla-La Mancha. https://ruidera.uclm.es/xmlui/bitstream/handle/10578/8674/TESIS\%20Jimeno\%20Jimenez.pdf?sequence=1\&isAllowed=y

Merino, C., \& Livia, J. (2009). Intervalos de confianza asimétricos para el índice de validez de contenido: Un programa Visual Basic para la V de Aiken. Anales de psicología, 25(1), 169-171. https://www.redalyc.org/pdf/167/16711594019.pdf

Merino-Martínez, R.M., Morillo-Gallego N., Sánchez-Cabezas A.M., Gómez-López V.E., \& Crespo-Montero, R. (2019). Relación entre la calidad de vida relacionada con la salud y la ansiedad/depresión en pacientes en hemodiálisis crónica. Enferm Nefrol, 22(3), 274-83. http://dx.doi.org/10.4321/S2254-28842019000300006

Ministerio de Salud del Perú. (05 de junio, 2014). Los trastornos mentales representan más de 32 años de vida saludables perdidos por discapacidad y muerte prematura por cada mil habitantes en el Perú [Comunicado de prensa]. http://www.insm.gob.pe/ oficinas/comunicaciones/notasdeprensa/2014/022.html 
Ministerio de Salud del Perú. (14 de febrero, 2020). Ministerio de Salud atendió más de un millón 200 mil casos relacionados a salud mental durante 2019 [Comunicado de prensa]. https://www.gob.pe/institucion/minsa/noticias/82187-ministerio-de-salud-atendiomas-de-un-millon-200-mil-casos-relacionados-a-salud-mental-durante-2019

Morales, C. (2017). La depresión: Un reto para toda la sociedad del que debemos hablar. Revista Cubana de Salud Pública, 43(2), 136-138. https://www.scielosp.org/pdf/ rcsp/2017.v43n2/136-138\#: :text=La\%20depresi\%C3\%B3n\%20provoca\%20un\%20 nivel,consiguiente $\% 20$ impacto $\% 20$ econ $\% \mathrm{C} 3 \% \mathrm{~B} 3 \mathrm{mico} \% 20 \mathrm{y} \% 20$ social

Organización Mundial de la Salud. (2004). Invertir en salud mental. https://www.who.int/ mental_health/advocacy/en/spanish_final.pdf

Organización Mundial de la Salud. (04 de abril, 2017). "Depresión: hablemos", dice la OMS, mientras la depresión encabeza la lista de causas de enfermedad [Comunicado de prensa]. http://www.paho.org/hq/index.php?option=com_content\&view=articl e\&id=13102\%3Adepression-lets-talk-says-who-as-depression-tops-list-of-causes-ofill-health\&catid $=740 \% 3$ Apress-releases\&Itemid=1926\&lang=es

Organización Mundial de la Salud. (mayo 2019). Salud mental en el lugar de trabajo [Comunicado de prensa]. https://www.who.int/mental_health/in_the_workplace/es/

Organización Mundial de la Salud. (30 de enero, 2020). Depresión [Comunicado de prensa]. https://www.who.int/es/news-room/fact-sheets/detail/depression

Organización Panamericana de la Salud (2017). Depresión y otros trastornos mentales comunes. Estimaciones sanitarias mundiales. http://iris.paho.org/xmlui/bitstream/ handle/123456789/34006/PAHONMH17005-spa.pdf

Ornelas Ramírez, A., \& Ruíz Martínez, A. O. (2017). Salud mental y calidad de vida: Su relación en los grupos etarios. PSIENCIA. Revista Latinoamericana de Ciencia Psicológica, 9(2), 1-16. https://doi:10.5872/psiencia/9.2.21

Pérez-Padilla, E., Cervantes-Ramírez, V., Hijuelos-García, N., Pineda-Cortés, J., \& Salgado-Burgos, H. (2017). Prevalencia, causas y tratamiento de la depresión Mayor. Revista Biomédica, 28(2) 73-98. https:/www.medigraphic.com/pdfs/revbio/bio2017/bio172c.pdf

Renner, F., Lobbestael, J., Peeters, F., Arntz, A., \& Huibers, M. (2011). Early maladaptive schemas in depressed patients: Stability and relation with depressive symptoms over the course of treatment [Esquemas desadaptativos tempranos en pacientes deprimidos: Estabilidad y relación con los síntomas depresivos a lo largo del tratamiento]. Journal of Affective Disorders, 136(3), 581-590. https://doi:10.1016/j.jad.2011.10.027

Ribeiro, F., dos Santos, P., Cazassa, M., \& da Silva Oliveira, M. (2014). Esquemas desadaptativos tempranos y síntomas depresivos: Estudio de comparación intergrupal. Revista Argentina de Clínica Psicológica, 23(1), 15-22. http://www.redalyc.org/articulo.oa?id=281943266003

Riso, R. \& McBride, C. (2007). Introduction: A return to a focus on cognitive schemas [Introducción: Un regreso a un enfoque en los esquemas cognitivos]. En L. Riso, P. du Toit, D. Stein, \& J. Young. (Eds.), Cognitive schemas and core beliefs in psycho- 
logical problems: A scientist-practitioner guide [Esquemas cognitivos y creencias fundamentales en problemas psicológicos: Una guía para científicos y profesionales]. (pp. 3-9). American Psychological Association.

Rivarola, A., Brizuela, M., \& Rolón-Ruiz, A. (2019). Nivel de depresión según la cronicidad de la Diabetes Mellitus tipo 2 y sus comorbilidades en pacientes de las Unidades de Salud. Salud Pública Paraguay, 9(2) 9-15. https://dx.doi.org/10.8004/rspp.2019. diciembre.9-15

Santelices, M. P., Guzmán, M., \& Garrido, L. (2011). Apego y psicopatología: Estudio comparativo de los estilos de apego en adultos con y sin sintomatología ansioso-depresiva. Revista Argentina de Clínica Psicológica, 20(1), 49-55. https://www.redalyc. org/articulo.oa?id=2819/281921807004

Vásquez, A. (2016). Los factores psicosociales y la depresión. Multimed. Revista Médica. Granma, 20(3), 586-602. https://www.medigraphic.com/pdfs/multimed/mul-2016/mul163j.pdf

Young, J. E., Schmidt, N., Joiner, Th., \& Telch, M. (1995). The schema questionnaire: Investigation of psychometric properties and the hierarchical structure of a measure of maladaptative schemas [El cuestionario de esquemas: Investigación de propiedades psicométricas y estructura jerárquica de una medida de esquemas desadaptativos]. Cognitive Therapy and Research, 19(3), 295-321.

Young, J. E., \& Klosko, J. S. (2001). Reinventa tu vida: Cómo superar las actitudes negativas y sentirse bien de Nuevo. Paidós

Young, J. E. (2005). Young Schema Questionnaire - Short form 3 (YSQ-S3) [Cuestionario de Esquemas de Young -Versión breve 3 (YSQ-S3)]. Cognitive Therapy Center.

Young, J.E., Klosko, J.S., \& Weishaar, M. (2013). Terapia de esquemas: Guía práctica. Desclée De Brouwer. 
